\title{
Self-consistent study of space-charge-driven coupling resonances
}

\author{
I. Hofmann* and G. Franchetti \\ GSI, 64291 Darmstadt, Germany \\ (Received 18 January 2006; published 30 May 2006)
}

\begin{abstract}
In this paper we present a comprehensive analysis of the self-consistent, collective behavior associated with the space-charge-driven (Montague) coupling resonance near $2 Q_{x}-2 Q_{y}=0$, including the effect of linear coupling. Based on analytical work and particle-in-cell simulation in the $2 \mathrm{D}$ coasting beam limit, we derive scaling laws for stop-band widths and growth rates, which may be applied to circular machines as well as to linear accelerators. For slow crossing of the stop bands, we find a strong directional dependence. In the case of crossing from below - assuming that the rising tune pertains to the direction, in which the initial emittance is the larger one-the emittance exchange is a smooth and fully reversible process. For crossing from above, we encounter a discontinuous behavior, which disappears largely, if an external linear coupling is applied.
\end{abstract}

DOI: 10.1103/PhysRevSTAB.9.054202

PACS numbers: 41.75. $-\mathrm{i}, 29.27 . \mathrm{Bd}, 29.17 .+\mathrm{w}$

\section{INTRODUCTION}

Emittance coupling due to space charge is known to be most effective near the fourth order resonance condition $2 Q_{x}-2 Q_{y}=0$. It was first analyzed in connection with circular accelerators in the frame of a single-particle approximation by Montague [1], with the conclusion that the resonance should be avoided by sufficient splitting of the tunes. In a number of operating or newly planned highcurrent synchrotrons, like the SIS-100 for the FAIR-project at GSI [2], tunes are not split by an integer. Hence, a detailed knowledge of the actual stop band is necessary to define an optimum working point with regard to highcurrent performance. For this reason, a detailed study of the Montague resonance was carried out at the CERN Proton Synchrotron in the years 2002 and 2003 [3]. It has opened the possibility of a comparison of theory with experimental data for bunched beams in a subject, where both space charge and nonlinear dynamics are closely interconnected. Such a benchmarking [4] is a complex undertaking, which eventually requires including 3D effects (synchrotron motion) and the fully nonlinear lattice of the accelerator.

The space-charge-induced emittance coupling is not only important for synchrotrons, but also for high-current linear accelerators as shown in Ref. [5]. There, the exchange can happen between the transverse and longitudinal degrees of freedom known as "equipartitioning."

For an in-depth theoretical understanding, it is important to realize that the Montague resonance is not just a singleparticle resonance phenomenon. The effect of space charge, instead, is evolving in time, which requires a self-consistent modeling. In order to explore the underlying collective phenomena several self-consistent particle-in-cell simulation studies have been carried out

*Electronic address: I.Hofmann@gsi.de primarily in $2 \mathrm{D}$, but also with some $3 \mathrm{D}$ aspects on a short time scale [6,7].

The present study is yet confined to the purely $2 \mathrm{D}$ coasting beam problem with the aim of offering a comprehensive treatment of the various linear and nonlinear features of this space-charge coupling resonance, including the important interaction with linear coupling. We present theoretical as well as practical aspects, including scaling laws and time scales. We proceed as follows: In Sec. II we give an overview of typical examples of emittance coupling in both constant and alternating gradient focusing, which is in part (constant focusing) reviewing earlier results . In Sec. III analytical scaling expressions for stopband widths and growth rates are compared with simulation results. Section IV is dedicated to the dynamical behavior for slow stop-band crossing and the question of reversibility. In Sec. V we discuss the modifications arising from the combined effect of the Montague resonance and additional linear coupling by adding skew quadrupoles to the lattice. Note that throughout the paper results are discussed in terms of the rms emittances (even if rms is not explicitly said); also, beams with different distribution functions are always understood as rms equivalent.

\section{BASIC FEATURES}

\section{A. Parameters}

In the following we define a "standard case" for a specific set of parameters, which has been typical for the experiments at the CERN proton synchrotron (PS) (ignoring synchrotron motion). Here it is worth noting that in the coasting beam case the physics can be described completely by three dimensionless quantities, the emittance ratio, the space-charge tune shift, and the relative position of the working point within the space-charge-induced stop band. For our standard example we have thus assumed a fixed vertical working point $Q_{0, y}=6.21$ and an emittance ratio of $\epsilon_{x} / \epsilon_{y}=3$, while the absolute values of initial 
normalized rms emittances are chosen as $\boldsymbol{\epsilon}_{x}=$ $2.5 \pi \mathrm{mm}$-mrad and $\epsilon_{y}=7.5 \pi \mathrm{mm}$-mrad. These units will be also used in all figures below. The current is set to yield a maximum vertical tune shift of $\Delta Q_{y}=0.105$ in the center of a Gaussian distribution. This leads to a maximum horizontal tune shift of $\Delta Q_{x}=0.061$ for the given emittance ratio. Results are obtained with the MICROMAP-code [8] employing 50.000 particles and a $128 \times 128$ grid with conducting boundary conditions on a square box of width 6 times the horizontal rms size of the beam.

Note that for applying the results of this paper to linear accelerator applications, where the coupling may occur between the transverse and longitudinal degrees of freedom, it is necessary to identify $x$ with $\perp$ and $y$ with $\|$, if $\epsilon_{\perp}>\epsilon_{\|}$; or $x$ with $\|$ and $y$ with $\perp$, if $\epsilon_{\|}>\epsilon_{\perp}$. Simulation examples supporting this analogy are given in Ref. [7].

\section{B. Time evolution}

The time behavior for different working points of the standard case under the assumption of a constant focusing and Gaussian input distribution is shown in Fig. 1. The rms emittance exchange (in units of $\pi \mathrm{mm}$-mrad in this and all following figures) increases, if $Q_{0, x}$ is chosen closer to $Q_{0, y}$. The rapid initial exchange is followed by emittance oscillations, which are slowly damped.

For tunes $Q_{0, x}$ very close to $Q_{0, y}$, the two emittances may shoot over their arithmetic mean. In Fig. 2 this occurs for $Q_{0, x}=6.207$ at turn 83 . The following emittance oscillations persist nearly undamped for thousands of turns. No damping at all is found for $Q_{0, x}=Q_{0, y}=6.21$, where a periodic emittance exchange is found-similar to that in a lattice with linear coupling from skew quadrupoles. For an uncoupled lattice as considered here this phenomenon is the "self-skewing" discussed in Refs. [7,9]: A spacecharge linear coupling force is induced by an-arbitrarily

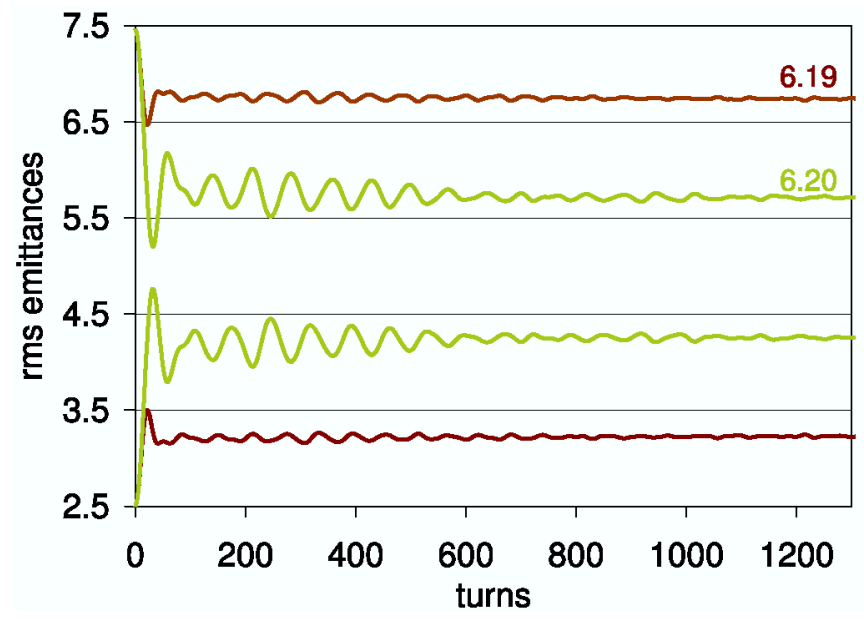

FIG. 1. (Color) Time evolution of rms emittances for $Q_{0, x}=$ $6.19,6.20$.

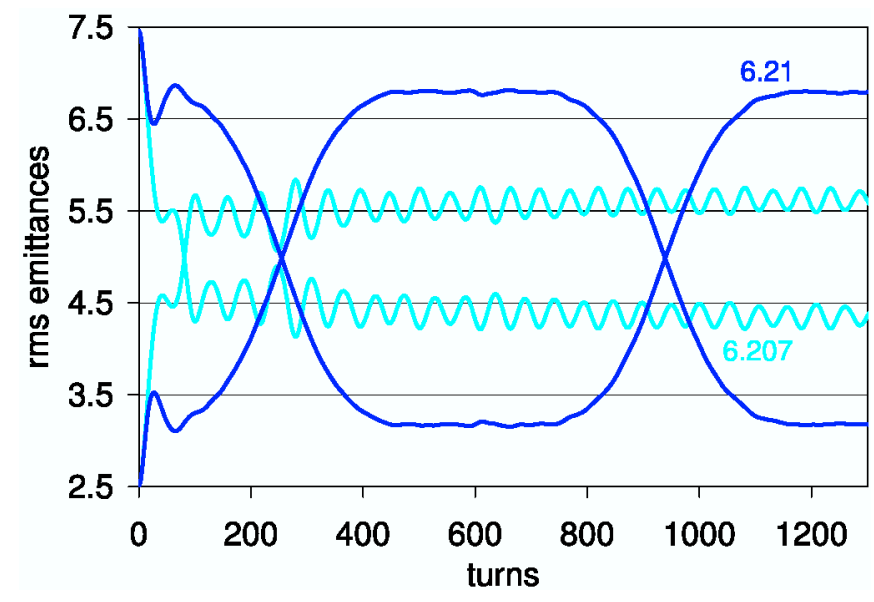

FIG. 2. (Color) Time evolution of rms emittances for $Q_{0, x}=$ 6.207 and $Q_{0, x}=6.21$.

small—initial beam rotation, which gets self-amplified in a resonance band (see also Sec. III A). The amplification is caused by exponential instability driven by space charge and the initial emittance imbalance.

A remarkable feature is the invariance of the sum of rms emittances, which is found constant within $\pm 10^{-3}$ for all cases studied here. With linear space charge and linear coupling, exact invariants are known to exist as was shown in Refs. [10,11]. It is not obvious why the nonlinear resonant processes present here keep the sum of emittances so highly invariant - an issue that requires further study.

\section{Simulation of stop bands}

In Fig. 3 we show the final rms emittances by varying $Q_{0, x}$ in small steps. The plotted values, where each marker is a simulation with different $Q_{0, x}$, are defined here and in all subsequent figures as averages of the rms emittance values between turn 1000 and 2000. This gives a good measure of the saturation stage, except for the case $Q_{0, x}=$ 6.21 , where there is no saturation.

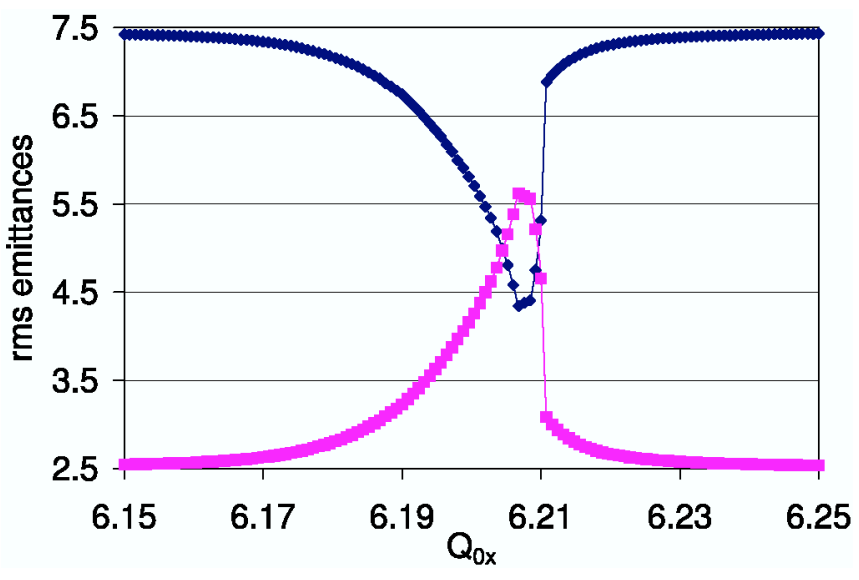

FIG. 3. (Color) Final rms emittances for variable $Q_{0, x}$ and constant focusing. 


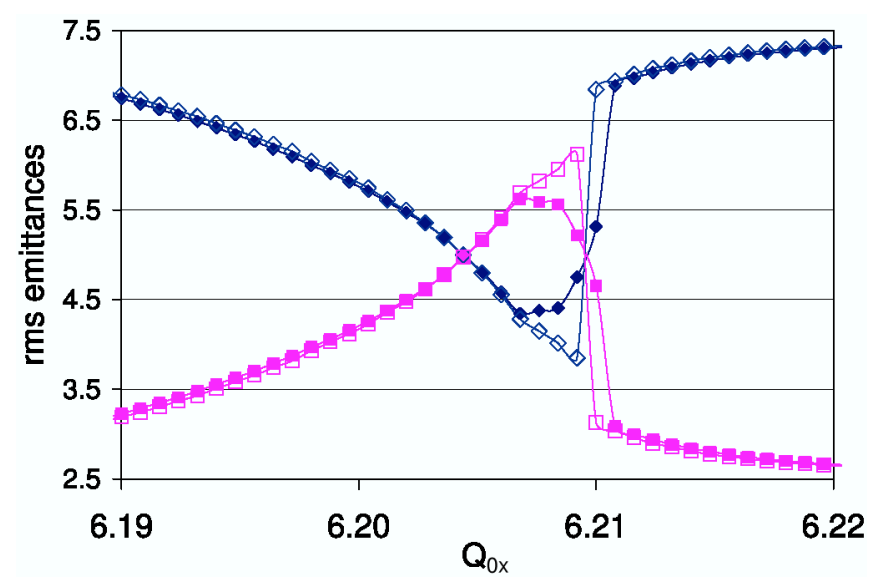

FIG. 4. (Color) Zoomed region comparing final emittances for constant (full markers) and periodic focusing (void markers).

In order to justify the use of constant focusing for the present study, we have compared it with (linear) periodic focusing as shown in Fig. 4, where the linearized alternating gradient lattice of the CERN PS was used. The difference is negligible almost everywhere in the stop band, which can be understood by the fact that what matters is not the instantaneous phase advance, but that averaged over many lattice periods. In a small region just below $Q_{0, x}=Q_{0, y}=6.21$, where the behavior is highly sensitive to tunes, we find that the overshoot is more pronounced for the periodic focusing case.

\section{SCALING LAWS}

\section{A. KV-beam reference case}

The specific differences between a waterbag and a Kapchinskij-Vladimirskij (KV)-distribution have already been discussed in Ref. [7]. A main point is that for non-KV distributions the fourth order space-charge potential driving the coupling is already present in the initial distribution, hence the emittance exchange starts immediately as seen in Figs. 1 and 2. For the KV-distribution, instead, the initial density is practically uniform, except for statistical fluctuations due to random initial seeds, which induce infinitesimally small space-charge multipoles. In Ref. [12] such small initial perturbations have been studied analytically using the linearized Vlasov's equation. As a result it was found that the noise-induced multipole oscillations may grow exponentially provided that emittances are unequal ("anisotropy" as "free energy" source) and that the tunes are sufficiently close to a resonance condition $n Q_{x}-m Q_{y}=0$, where $|n|+|m| \leq 4$. The unstable eigenmodes are driving the emittance exchange. Hence, for initial KV-beams the "Montague resonance" also exists, but with the modification that nonlinearity and resonant coupling start entirely from initial noise with exponential self-amplification by instability. In fact, the stop-band widths and the quantitative amount of emittance

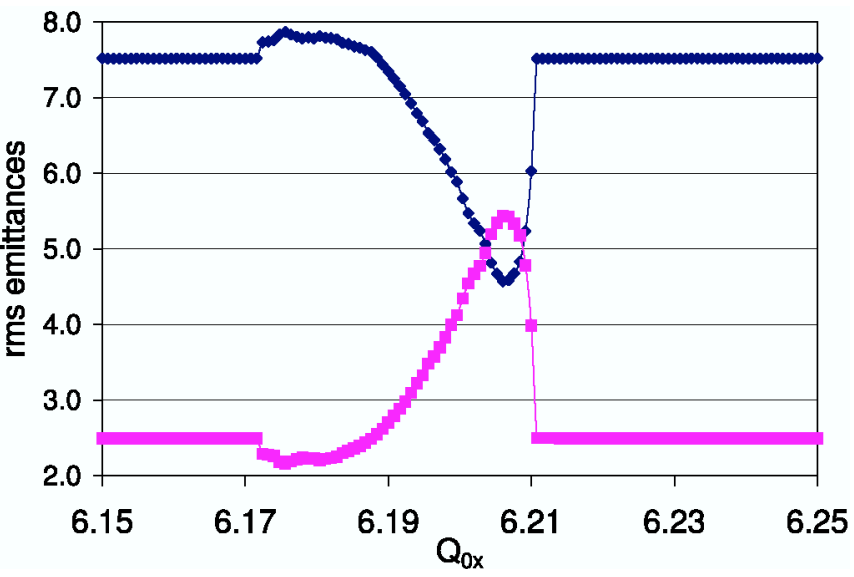

FIG. 5. (Color) Final rms emittances for KV-distribution and constant focusing.

exchange are very similar as is shown in Fig. 5 for constant focusing.

This suggests that the analytical Vlasov theory results for KV-beams may be considered as a basis for deriving scaling laws for stop-band widths of more general distributions.

Before doing so, we compare the findings of Fig. 5 with the analytical Vlasov theory. For modes up to fourth order, where "order" describes the power of the terms in $x, y$ in the perturbed space-charge potential, the growth rates of different modes can be calculated as function of tunes by using the dispersion polynomials of Ref. [12]. There, the exponential growth rates are expressed in units of betatron tunes as $\Im \omega / Q_{0, y}$. Results are shown in Fig. 6 for constant focusing and using the parameters of Sec. II A. For convenience - to facilitate application to rings as well as linacs-we express the calculated rates as inverse of the number of (zero space charge) betatron periods, $N_{\beta}$, required to obtain one e-folding of amplitude growth by using the relationship

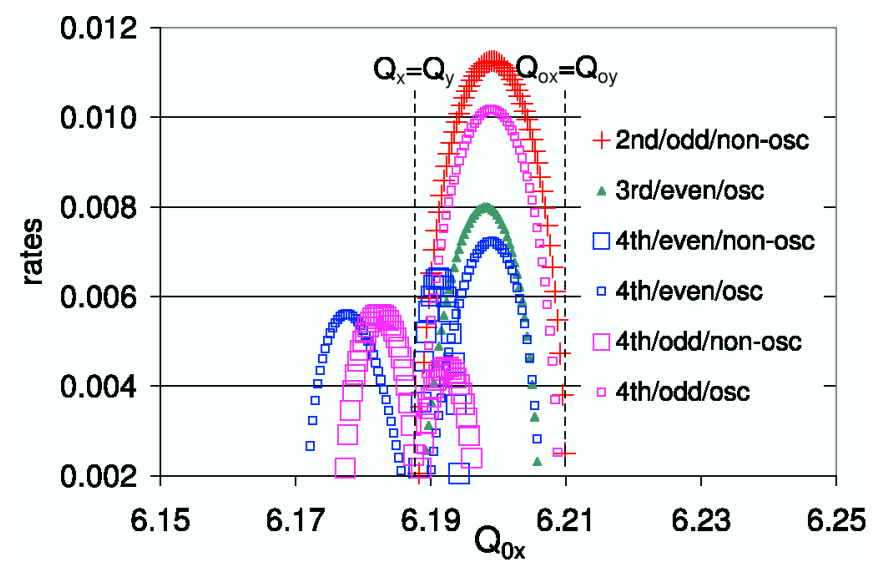

FIG. 6. (Color) Growth rates $N_{\beta}^{-1}$ for collective modes of KVdistribution. 


$$
N_{\beta}^{-1} \equiv 2 R \pi \frac{\Im \omega}{Q_{0, y}},
$$

where $2 R \pi$ is the ring circumference, or, more generally, the distance over which the tune is defined. In each order "even" and "odd" modes must be distinguished, relating to the $x-y$ symmetry: in second order the even modes are envelope oscillations (with terms $x^{2}$ and $y^{2}$ in the spacecharge potential), whereas the odd modes are linear coupling modes (with terms $x y$ ), and similar in higher orders. Furthermore, the unstable modes can be classified in two categories distinguished by different size markers: small markers relate to oscillatory $\mathrm{KV}$-instabilities $(\operatorname{Re} \omega \neq 0)$, larger markers to nonoscillatory instabilities $(\operatorname{Re} \omega=0)$. In Ref. [7] it is postulated and confirmed by a set of simulations that the oscillatory instabilities are an artifact of the $\mathrm{KV}$-distribution, which is absent for monotonically decreasing distribution functions. The nonoscillatory instabilities, however, are of concern for all distributions, whether KV or non-KV. Hence, the oscillatory solutions in Fig. 6 should be ignored whenever monotonically decreasing distribution functions-like waterbag or Gaussians - are considered, which is the case here. For completeness we point out that "nonoscillatory" is related here to the observation that the initial collective perturbation is purely growing, and collective oscillation-at the scale of the relatively fast betatron frequencies - is absent. The applicability of $N_{\beta}$ as measure for the time needed for emittance exchange, which is part of the nonlinear rather than the linear evolution, is deferred to Sec. III C.

The full stop-band width suggested from the region of nonvanishing growth rates in Fig. 6 is in excellent agreement with the simulation stop-band width of Fig. 5. It is also noted that in Fig. 6 all growth rates vanish at the point $Q_{0, x}=6.188$, where-for our parameters - the condition $Q_{x}=Q_{y}$ holds. This agrees with the absence of emittance exchange at the same point in Fig. 5. It is a peculiarity of the KV-case that for tunes smaller than this value the emittance exchange is in the "wrong" direction, i.e., the final emittances are even more apart than the initial ones. This "antiequipartition" is only found for the KVdistribution; it appears to be connected with the left branch of the oscillatory fourth order even mode in Fig. 6, which is a so-called "negative energy oscillation" [12]. We also notice in Fig. 6 that for values of $Q_{0, x}$ beyond the point $Q_{x}=Q_{y}$ a second order odd (nonoscillatory) mode instability exists, which extends exactly up to the point, where $Q_{0, x}=Q_{0, y}$. Note that in this "self-skewing band" the condition $Q_{x}>Q_{y}$ and simultaneously $Q_{0, x}<Q_{0, y}$ applies, which expresses that the direction of stronger applied focusing is reversed by space charge. The self-skewing instability was already discussed in the simulations of Sec. II B, where it was, however, observed only at the point $Q_{0, x}=Q_{0, y}$.
Based on this reasoning we define an "effective stop band" from the analytical theory by considering only the region, where the nonoscillatory modes have nonvanishing growth rates. Our simulations, carried out for a diversity of emittance ratios and intensities, fully confirm that the sodefined stop band describes the tune range, where Gaussian as well as waterbag beams show emittance coupling. We can, therefore, make use of the analytical theory to derive scaling laws for stop-band widths applicable to general beam distributions, which will be presented in the following section (and similar for the growth times in Sec. IIIC).

\section{B. Stop-band width scaling}

In spite of the high complexity of the algebraic expressions for dispersion relations of the coupling modes, in particular, in fourth order, we have found from numerical solutions of the dispersion polynomials that surprisingly simple scaling laws exist for the stop-band widths. For calculating the width of the region, where the nonoscillating $\mathrm{KV}$-modes have finite growth rates (the effective stop band), we proceed as follows. Here we find it convenient with reference to both ring and linac applications-to express the stop-band width in terms of the ratio of space-charge depressed tunes as variable. As a useful scale for the required full stop-band width we take the width of the above introduced "self-skewing band," which is easily expressed in this variable as

$$
\delta \equiv\left[Q_{x} / Q_{y}\right]_{Q_{0, x}=Q_{0, y}}-1 .
$$

This is easily rewritten by introducing the relative tune shifts $\widetilde{\Delta Q_{y}} \equiv \Delta Q_{y} / Q_{0, y}$ for normalization:

$$
\tilde{\delta} \equiv \frac{\delta}{\widetilde{\Delta Q_{y}}}=\frac{\sqrt{\epsilon}-1 r}{\sqrt{\epsilon}\left(1-\widetilde{\left.\Delta Q_{y}\right) r}\right.}
$$

Here we have used $\epsilon_{r} \equiv \epsilon_{x} / \epsilon_{y}$ and the relationship

$$
\Delta Q_{y} / \Delta Q_{x}=\sqrt{\epsilon_{r}} .
$$

Figure 6 suggests that the above introduced effective stop band has a width, which is about 1.5 times that given by Eq. (2). In order to check whether such a factor also applies to other choices of parameters, we introduce it as $g_{w}$ in the expression for the "effective stop-band" width, e.g.

$$
\tilde{\delta}_{\text {eff }}=g_{w} \frac{\sqrt{\epsilon}-1 r}{\sqrt{\epsilon}\left(1-\widetilde{\left.\Delta Q_{y}\right)}\right)} .
$$

For the results shown in Fig. 7 we have first determined $\tilde{\delta}_{\text {eff }}$ by solving the dispersion relations as in Fig. 6. Using Eq. (5) we have then calculated $g_{w}$, which is plotted for a wide range of emittance ratios as well as relative tune shifts. The latter are indicated by the value of $\widetilde{\Delta Q_{y}}$. Note that the smaller values of $\widetilde{\Delta Q_{y}}$ relate to circular machines, and the larger values to linear accelerator applications. 


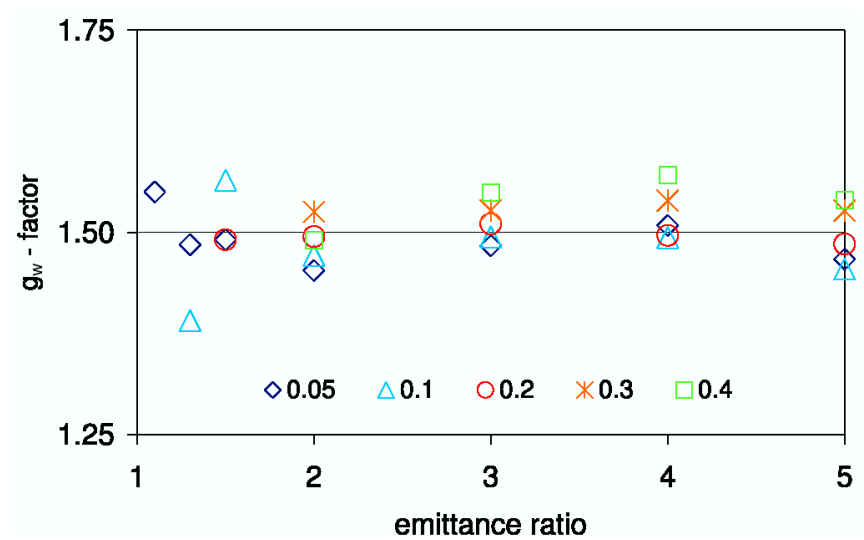

FIG. 7. (Color) Numerical results for $g_{w}$ as a function of emittance ratio, and for different values of (relative) space-charge tune shift.

This confirms that $g_{w}=3 / 2$ can be used as a universal value describing the effective extent of the stop band to the left of the point, where $Q_{0, x}=Q_{0, y}$. We assume that the jitter of $g_{w}$ in Fig. 7 is related primarily to rounding errors in determining solutions of the high order polynomials in the dispersion relations, especially for $\epsilon_{r} \rightarrow 1$.

For small tune shifts, as in circular machines, it is convenient to replace the width in the tune ratio by the equivalent width, $\Theta$, on the scale $Q_{0, x}$. Using the relationship

$$
\tilde{\delta}=\frac{\Theta}{\Delta Q_{y}},
$$

we may rewrite Eq. (5) in the form

$$
\Theta_{\text {eff }}=\frac{3}{2}\left(\sqrt{\epsilon_{r}}-1\right) \Delta Q_{x} .
$$

We note that $\Delta Q_{x}$ is introduced here as space-charge tune shift of an equivalent KV-beam, which is half the maximum tune shift of a Gaussian beam. Also, $\epsilon_{r}>1$ is understood here; if $\epsilon_{r}<1$ Eq. (7) still holds if $x$ is consistently replaced by $y$.

To illustrate the applicability of these analytical results to the particle-in-cell simulations, we observe that Eq. (7) predicts for the case of Fig. 3 the left edge of the stop band at 6.177. This agrees with the extent of the region of "significant" exchange, if we disregard the points, where $\leq 5 \%$ of the maximum possible exchange occurs. The agreement is found equally good for other parameters.

\section{Growth rate scaling}

By using the dispersion polynomials of Ref. [12], we have calculated numerically the maximum theoretical mode growth rates $N_{\beta}^{-1}$ (shown in Fig. 6 for one example) for the same set of parameters as in Fig. 7. We have found that these rates follow the same trend in their dependence on $\Delta Q_{x}$ and $\epsilon_{r}$ as the stop-band widths and therefore postulate a similar scaling expression

$$
N_{\beta}^{-1}=2 \pi g_{r}\left(\sqrt{\epsilon_{r}}-1\right) \frac{\Delta Q_{x}}{Q_{0, x}} .
$$

For the proportionality factor $g_{r}$, we find that the value $1 / 2$ provides a good fit, which is accurate to within less than $5 \%$ deviations for the whole range of parameters considered. Note that, as introduced in Sec. III A, $N_{\beta}$ is understood here as number of betatron periods needed to complete one e-folding of the underlying eigenmode amplitude. We find that a case distinction between large and small $\Delta Q_{x}$, as in Eqs. (5) and (7), for the stop-band width, is unnecessary here.

As mentioned in Sec. III A, a direct comparison of these eigenmode growth rates with an actual "emittance exchange rate" for non-KV-beams is conceptually not straightforward. The mode growth rates result from small perturbation Vlasov theory, hence there is exponential growth, whereas the emittance exchange of Gaussian beams occurs largely in a nonlinear stage - due to the already very nonuniform initial density profile-which leads to a more linear time dependence. In order to evaluate the latter from simulations we define a simulation based emittance exchange rate, $N_{\mathrm{ex}}$, in the following way: we determine the number of betatron periods, $\Delta N_{\min }$, where the first minimum of $\epsilon_{x}$ occurs with a corresponding reduction by $\Delta \epsilon_{\min }$, calculate the corresponding slope, and normalize it on half of the initial emittance difference, hence

$$
N_{\mathrm{ex}}^{-1} \equiv \frac{2 \Delta \epsilon_{\min }}{\Delta N_{\min }\left(\epsilon_{x}-\epsilon_{y}\right)}
$$

In Fig. 8 these rates are shown as function of $Q_{0, x}$ for the case of Fig. 3.

The maximum of this rate is 0.00467 at the tune $Q_{0, x}=$ 6.20 , which corresponds to 214 betatron periods, or 34 turns for full emittance equalization (equipartition). As far as the dependence on the space-charge tune shift we have found from simulation runs at different intensities that

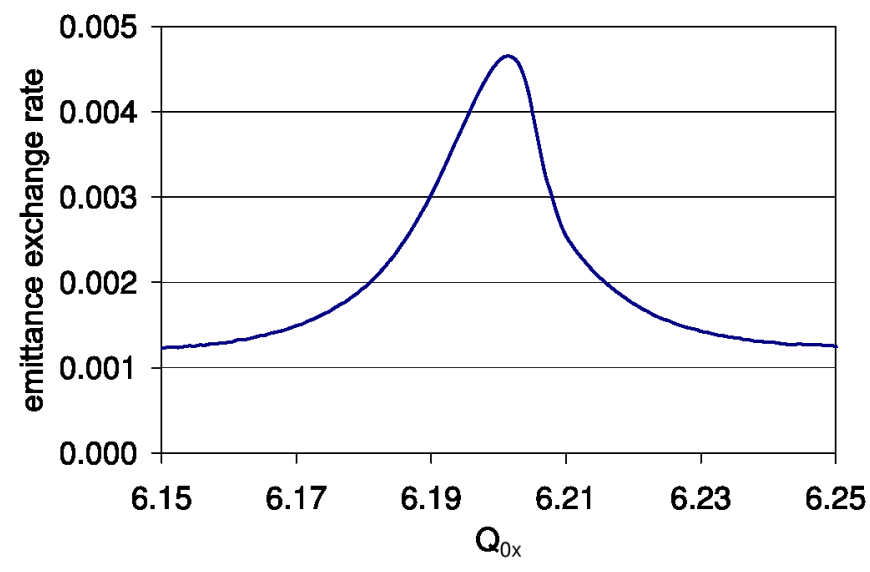

FIG. 8. (Color) Simulation result for normalized "emittance exchange rates" as a function of $Q_{0, x}$ and $\epsilon_{r}=3$ 
$N_{\text {ex }}^{-1} \propto \Delta Q_{x} / Q_{0, x}$ holds. Also, $N_{\text {ex }}$ depends only on the emittance ratio, $\epsilon_{r}$, and not on the absolute value of emittances. The dependence on $\epsilon_{r}$ in Gaussian beam simulations is actually found weaker than predicted by Eq. (8). In the range $1.5 \leq \epsilon_{r} \leq 3$ a good fit to the simulation results is given by the simple expression, where $\Delta Q_{x}$ is referring to the equivalent $\mathrm{KV}$-beam tune depression:

$$
N_{\mathrm{ex}}^{-1} \approx \frac{\Delta Q_{x}}{Q_{0, x}} .
$$

In a high-current linac with $50 \%$ tune depression by space charge, for example, hence $\Delta Q_{x} / Q_{0, x}=0.5$, this suggests that the fastest exchange requires two (undepressed) betatron periods only.

\section{DYNAMICAL CROSSING OF RESONANCES}

In the previous sections we have explored emittance coupling for fixed working points and found that Gaussian beams settle down to a new equilibrium, where the final emittances have come closer to each other (except for the periodic exchange for $Q_{0, x}=Q_{0, y}$ ). This "static" case is substantially different from the case, where emittance equalization is achieved by slowly moving the tune across the resonance - the "dynamical" case.

\section{A. Reversible and irreversible emittance exchange}

For dynamical crossing we use the standard case of Fig. 3 and move the working point $Q_{0, x}$ starting from the side of lower tunes over the range $6.15 \leq Q_{0, x} \leq 6.27$ enclosing the stop band. For this crossing "from below" we apply a linear tune ramp in time. In Fig. 9 we show the evolution of emittances as function of the instantaneous tune for two cases, where the crossing of the same tune range is performed in 100, respectively, 1000 turns.

It is noted that for the 100 turns case the final emittances are practically equal; for the 1000 turns case the final

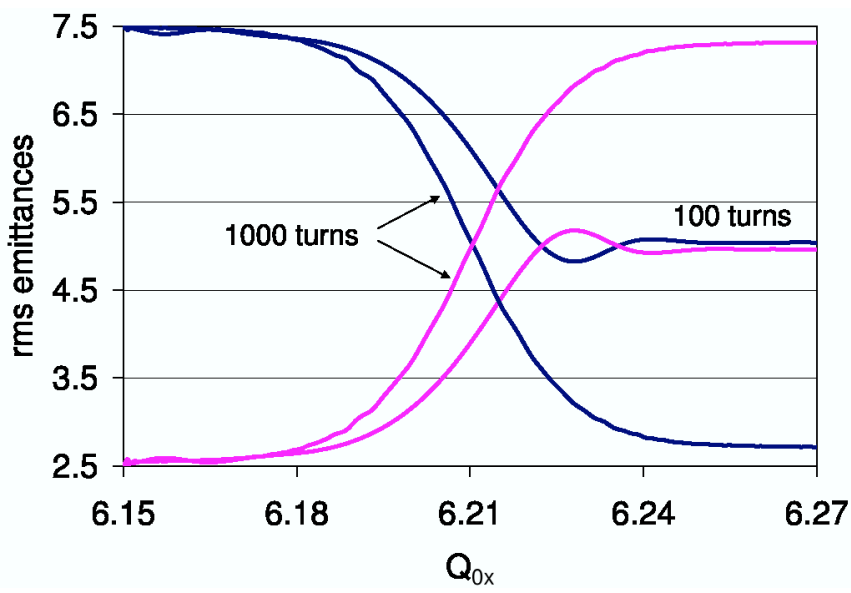

FIG. 9. (Color) Evolution of emittances by crossing the stop band dynamically from below over 100 and 1000 turns.

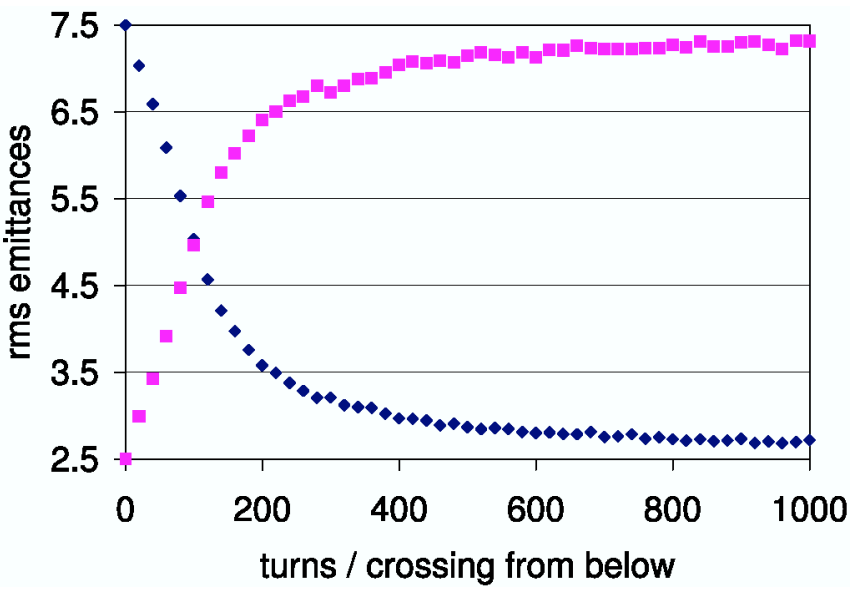

FIG. 10. (Color) Final emittances after crossing $Q_{0, x}=6.15 \rightarrow$ 6.27 at variable rates.

emittances are basically exchanged with the initial emittances.

A more complete picture of the final result after crossing the band at variable ramp times is shown in Fig. 10.

Note that the 100 turns case, where the final emittances result as equal, is a dynamically achieved equipartition. In this case the essential part of the stop band in Fig. 3, which has a tune width of 0.04 , is crossed in 33 turns or 205 betatron periods. This time agrees with the fastest rise time of the static case, which therefore sets the time scale needed for a crossing to just equalize final emittances. Note that for faster crossing the exchange is only partial, and for slower crossing we get the reversal of final emittances. We may call the resonance crossing "adiabatic," if the final emittance exchange is close to a full reversal, i.e., the final $\epsilon_{x}$ is close to the initial $\epsilon_{y}$ and vice versa. We have found that this requires a crossing time, which is about an order of magnitude longer than that for dynamical equipartition. Hence, the number of betatron periods needed to satisfy the adiabaticity condition can be approximately written as

$$
N_{\text {ad }} \gg N_{\text {ex }},
$$

if $N_{\text {ex }}$ is the fastest growth of the static case as given by Eq. (10).

Another aspect of the slow, adiabatic crossing is a kind of "snow-plow" effect discussed also in Ref. [9] in the context of pure linear coupling. The idea is that when $Q_{0, x}$ reaches the stop-band edge, it always remains at the edge of it. In other words, the emittances self-consistently move closer to each other in such a way that the edge of the shrinking stop band coincides with $Q_{0, x}$, until the point $Q_{0, x}=Q_{0, y}$ is reached. There, the emittances are equal, hence the stop-band width is zero following Eq. (7). This snow-plow effect is thus a direct consequence of the emittance-dependent detuning curve described by Eq. (7). In fact, we can retrieve to good accuracy the emittance 
coupling shown in Fig. 9 by simply resolving Eq. (7) for $\epsilon_{r}$, noting that $\Delta Q_{x}$ also implicitly depends on $\epsilon_{r}$.

Here it may be asked, where the memory of the initially unequal emittances is stored, when the rms emittances are equalized at $Q_{0, x}=6.21$, but return to the inverted ratio for $Q_{0, x}>6.21$. Obviously, the phase space distribution at $Q_{0, x}=6.21$ cannot be that of an equipartitioned Gaussian, for which theory predicts full absence of any resonant exchange. By inspecting various projections into coupled phase planes we find that a strong correlation with fourfold symmetry exists in the $x-y^{\prime}$ plane (similarly in the $y-x^{\prime}$ plane), which reflects the nature of the underlying octupolar space-charge coupling and the initial emittance imbalance. This is shown in Fig. 11, where the comparison with the 100 turn crossing gives evidence for the absence of this clear correlation due to the faster crossing.

In fact, our study suggests that for this slow crossing the phase space distribution is continuously matched to the underlying nonlinear Hamiltonian at all tune values, if it was initially matched for a tune far from the resonance. The nonlinear Hamiltonian here is the superposition of the linear lattice part and the self-consistent nonlinear spacecharge potential. For completeness we note that this fourfold correlation is entirely absent, if the initial emittances are chosen equal.

The above described "adiabatic crossing" is also a reversible process, in spite of the nonlinear nature of the underlying motion. To verify this quantitatively we have used the output of the simulations of Fig. 10 for crossing from below, and crossed the resonance a second time from above, by following the mirrored tune ramp $Q_{0, x}=6.27 \rightarrow$ 6.15 with the same number of turns as in the upwards crossing. Results shown in Fig. 12 confirm the reversibility of the first crossing process, if the crossing is sufficiently slow. Very fast crossing causes only small emittance changes both ways. Crossing with the above defined rate of dynamical equipartition shows the highest degree of "irreversibility"-emittance equipartition is reached in the crossing from below and maintained in the subsequent crossing from above. Some caution is necessary here as far as the notion of irreversibility and equipartition is con-
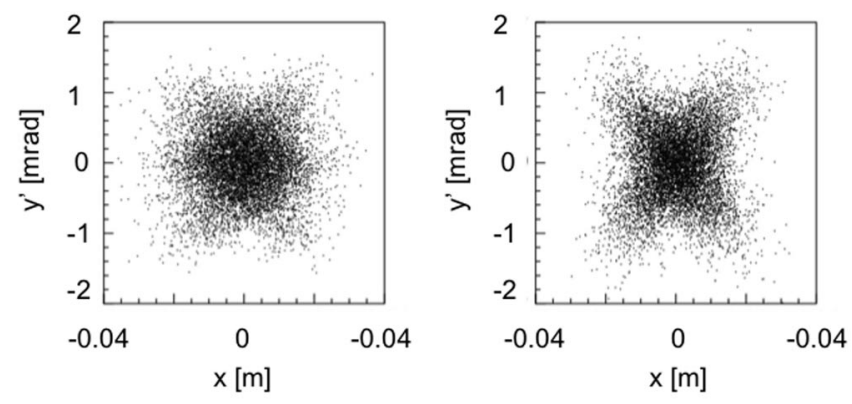

FIG. 11. Projection onto coupled phase planes at $Q_{0, x}=6.21$ for 100 (left frame) and 1000 (right frame) turn crossing.

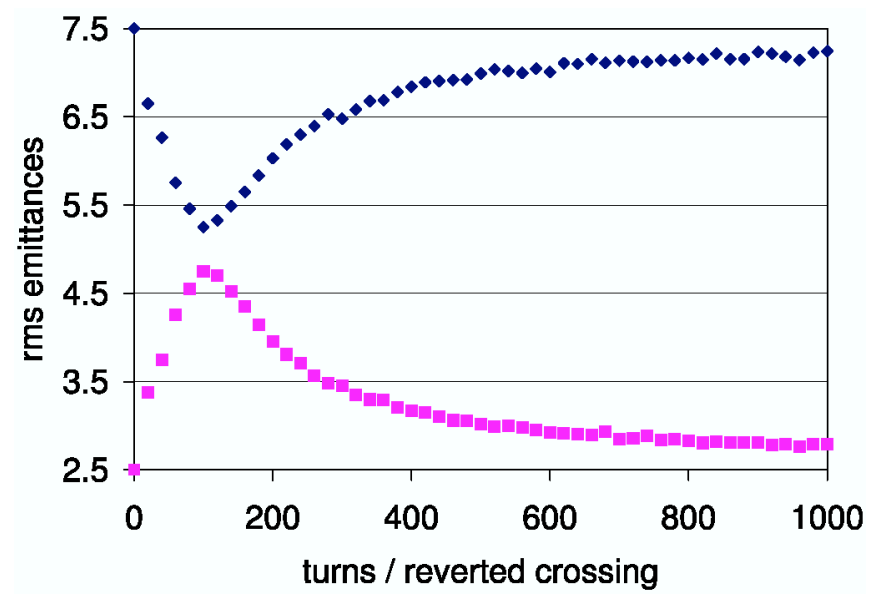

FIG. 12. (Color) Final emittances after double crossing with $Q_{0, x}=6.15 \rightarrow 6.27 \rightarrow 6.15$ at a variable number of turns (related to one direction).

cerned. Here, equipartition does not imply that a thermodynamic equilibrium state or real Gaussian distribution has been reached. Likewise, we are not claiming that individual particle orbits are necessarily chaotic and orbit timereversibility - if velocities were reversed in addition to the tune change - is violated. Our simulations only support that macroscopic phase space correlations are destroyed, which would allow retrieving the initial rms emittance imbalance - a property of second order moments.

\section{B. Crossing the resonance "from above"}

The nonlinear nature of the underlying collective spacecharge effect is strongly evidenced, if the stop band is crossed from above by using the original emittances in $x$ and $y$. This is shown in Fig. 13 for otherwise the same parameters as in Fig. 9. Note that crossing from above, but with reversed initial emittances, would give the curves of Fig. 9 just mirrored.

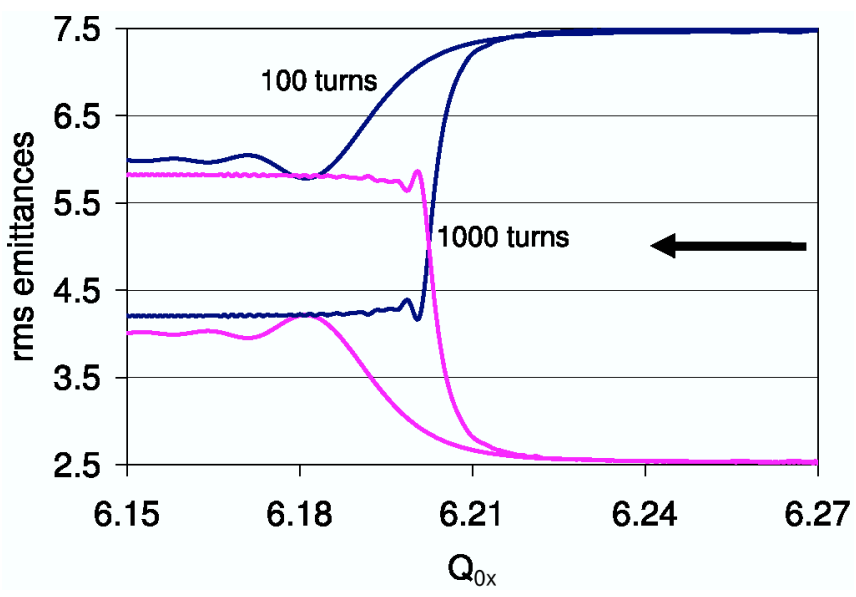

FIG. 13. (Color) Crossing from above at two different rates (100 and 1000 turns). 
The slower crossing does not lead to a full exchange. This behavior reflects the very sharp gradient near $Q_{0, x}=$ 6.21 as seen in Fig. 4. The 1000 turns case corresponds to a change in tune of 0.005 within about 40 turns, hence this crossing passes through the "overshoot" region between 6.205 and 6.21 in a time, which is comparable with the fastest growth in this region according to Fig. 8. A 10 times slower crossing (10.000 turns for the same tune span as in Fig. 13) shows in Fig. 14 - at first glance surprisinglyincreasing oscillations, which are washed out further away from the resonance. This behavior can also be understood in the light of Fig. 4: in about 80 turns a tune change of 0.001 occurs, which is approximately the width of the narrow region below $Q_{0, x}=6.21$, where pure self-skewing is found in the static tune case. Hence, we may assume that the full emittance exchange oscillation seen in the simulation has been picked up from the instability of the selfskewing mode. Contrary to the crossing from below, we see no indication that the solution follows that of a matched nonlinear Hamiltonian.

For a still slower crossing, within 50.000 turns as shown in Fig. 15, we find that similar emittance oscillations exist. The first crossover of emittances is reached even closer to $Q_{0, x}=6.21$ - the system penetrates less into the unstable parameter region. Although growth rates of the unstable self-skewing mode are getting the smaller the closer $Q_{0, x}$ is to 6.21 , the achieved growth is compensated by the effect of a much longer time available for the growth. Also, the final emittance exchange is coming closer to $100 \%$. We may assume that for arbitrarily slow crossing the first crossover point moves asymptotically towards $Q_{0, x}=$ 6.21 , and the emittance exchange reaches the $100 \%$ level.

This behavior is consistent with the observation that for $Q_{0, x}>6.21$ an emittance ratio $\epsilon_{x}>\epsilon_{y}$ is stable, whereas for $Q_{0, x}<6.21$ the ratio $\epsilon_{x}<\epsilon_{y}$ is stable. With the crossing from above the solution just "flips" from the unstable solution to a stable one (with inverted emittance ratio) by

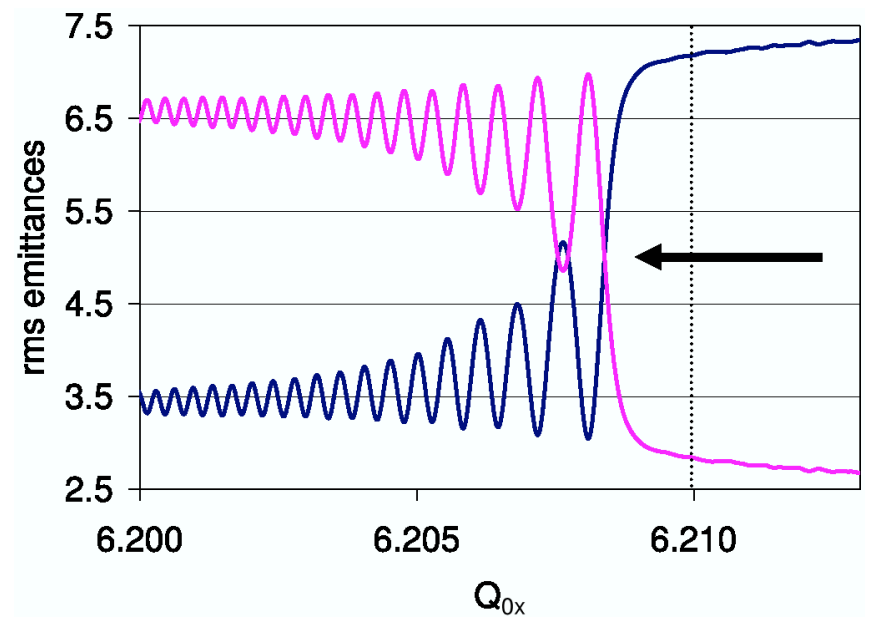

FIG. 14. (Color) Crossing from above (zoomed tune region) at 10.000 turns.

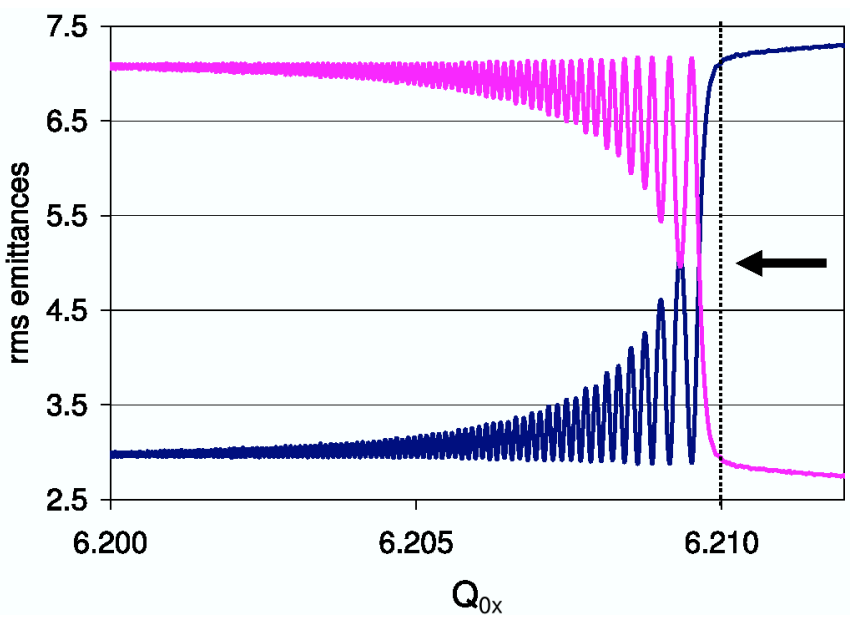

FIG. 15. (Color) Crossing from above (zoomed tune region) at 50.000 turns.

means of the self-skewing instability. More precisely, it performs an oscillation about the new stable solution, which is damped, while the tune moves further away from the resonance. Hence, once the working point enters the stop band from above it is "attracted" by it: the stop band quickly flips to the other side of $Q_{0, x}=6.21$ due to the self-consistent emittance exchange, and the resonant interaction ceases. A similar behavior was obtained in a study of pure linear coupling with space charge in Ref. [9]. There, in the example of unsplit tunes (with skew quadrupoles absent), the self-skewing mode was the only possible source of coupling due to the use of purely second order moment equations describing the beam.

Note that this behavior reflects the fact that the position of the right edge of the stop band (for the assumed ratio $\epsilon_{x}>\epsilon_{y}$ ) is just given by $Q_{0, x}=Q_{0, y}$, independent of the emittance ratio. For the left edge, instead, we have the emittance-dependent detuning, which leads to the above discussed snow-plow effect. It implies that this edge of the stop band is actually "repulsive" rather than "attractive," since the tune effectively always remains at the edge.

\section{Scaling invariance with intensity}

We find that all graphs of Sec. IV can be directly scaled to any other value of $\Delta Q_{x}$, if the number of turns for stop band crossing is properly adjusted. Multiplying $\Delta Q_{x}$ by a factor $\alpha$, the graphs are invariant if the band over which the tune is swept is also expanded by $\alpha$ and the number of turns for crossing - now related to sweeping over the expanded band - is divided by $\alpha$, hence the tune rate (change of tune per turn) is multiplied by $\alpha^{2}$. This is a direct consequence of the linear scaling of both the stop-band width and the growth rate, with $\Delta Q_{x}$. Defining $\dot{Q}$ as tune change per turn, we can introduce a normalized number of turns,

$$
\tau \equiv \frac{\left(\Delta Q_{x}\right)^{2}}{\dot{Q}}
$$


assuming that the full stop band is crossed at a constant rate. Hence, two crossings with different rates and intensities lead to the same emittances, if they agree in $\tau$. Note that in Figs. 10 and 12 the unit of 100 turns-when equipartition is reached-corresponds to $\tau=3$. Equation (12) can be also resolved for $\dot{Q}$. For our linac example with $\Delta Q_{x} / Q_{0, x}=0.5$ this implies that for just equipartition a tune ramp is needed, which corresponds to a relative change of focusing of 0.08 .

\section{LATTICE WITH LINEAR COUPLING}

Adding linear coupling by skew components in the lattice leads to further modifications. Obviously, the emittance exchange is skew-dominated, if the stop-band width due to pure linear coupling - with the resonance condition $Q_{0, x}-Q_{0, y}=0$ - exceeds that of the Montague resonance. Here we are interested in the opposite case of large space-charge effects, which is the more likely one in highcurrent accelerators. For the discussion of the space-charge effects on linear coupling in a purely second order approximation-suppressing the fourth order Montague resonance - we refer to Ref. [9].

\section{A. Static stop bands}

Similar as in Ref. [9], we define the strength of linear coupling by the number of turns needed for the first complete emittance exchange in the absence of space charge, $N_{s}$, which we assume here to be 200 . The skew is applied here by a single kick per turn. For the sake of clarity we show in Fig. 16 the familiar result for "final" rms emittances, defined here again as averages as in Sec. II C. The actual stop-band width (FWHM) is $\approx 0.005$ in our example ( $\approx 15 \%$ of that of the Montague resonance), hence we are in the limit of weak skew relative to space charge.

Combining space charge with the so-defined skew, we obtain a modification of Fig. 3 as shown in Fig. 17, which indicates a clearly split picture: The very sharp gradient is

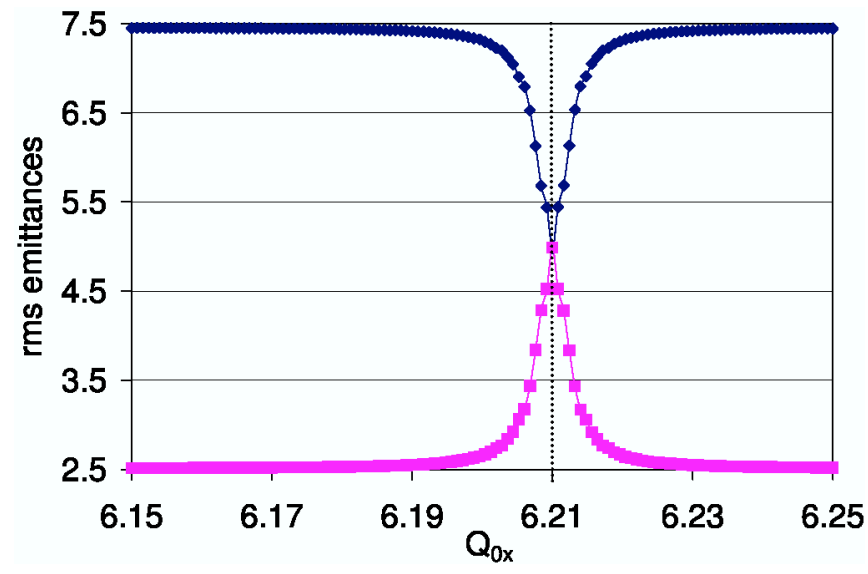

FIG. 16. (Color) Final rms emittances for variable $Q_{0, x}$ with only linear coupling (no space charge).

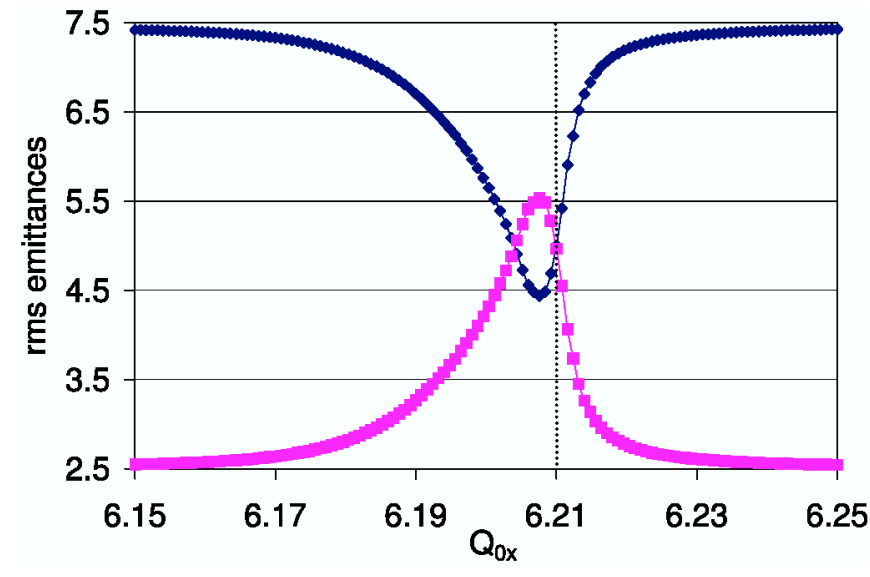

FIG. 17. (Color) Final rms emittances for variable $Q_{0, x}$ with space-charge and linear coupling.

replaced above $Q_{0, x}=6.21$ by the skew-specific exchange nearly identical to Fig. 16, whereas below $Q_{0, x}=6.21$ we retrieve the response as in the absence of skew. Note that the left edge of the stop band is hardly influenced by the skew, which is due to the fact that the Montague resonance dominates over the effect of skew, except for the region above $Q_{0, x}=6.21$.

\section{B. Dynamical crossing}

This behavior has an impact on dynamical crossing. We first show results in the absence of space charge in Fig. 18. It is expected that for effective emittance exchange the actual linear coupling stop band should be crossed in more than $N_{s}$ turns. In our example, the linear coupling stop band is 5\% of the scanned tune window, hence effective emittance exchange would require that this window is crossed in more than 4000 turns. This is well confirmed by Fig. 18, where the exchange is nearly complete with 5000 turns, while a 1000 turn crossing hardly reaches equipartition (crossing in 100 turns makes $\epsilon_{x}$ drop by

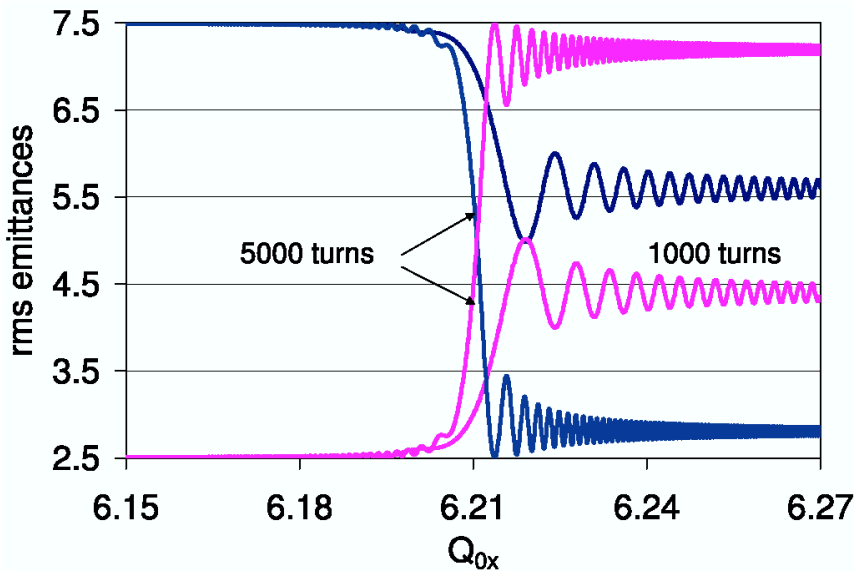

FIG. 18. (Color) Evolution of emittances by dynamical crossing over 1000 and 5000 turns, only linear coupling (no space charge). 


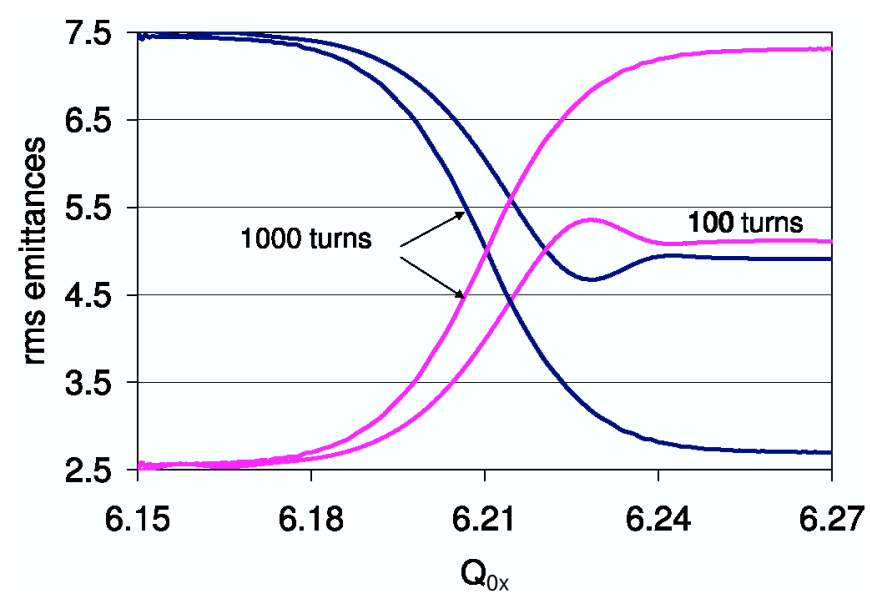

FIG. 19. (Color) Dynamical crossing from below over 100 and 1000 turns, linear coupling, and space charge.

less than 10\%). Note that the direction of crossing is irrelevant without space charge.

Including space charge, we show in Fig. 19 that the crossing from below is only very weakly modified by the skew (compare with Fig. 9). This is fully consistent with the snow-plow effect, if we note that the left edge of the stop band is actually controlled by the effect of space charge and not by the skew.

For crossing from above we find a different result. The split response on the right edge of the stop band has the effect that the exchange starts as a result of the skew effect and space charge enhances it, in fact the combined effect of skew and space charge exceeds by far the coupling efficiency of either one. In Fig. 20 it is seen that even the fast crossing in the 100 turns case leads to almost equipartition, whereas without space the exchange (not shown here) was found below 10\%. The 10 times slower crossing (1000 turns) gives almost a full exchange of emittances due to the space-charge enhancement of the pure skew effect. For much slower crossing from above, the exchange is entirely controlled by the skew effect, which then justifies applying

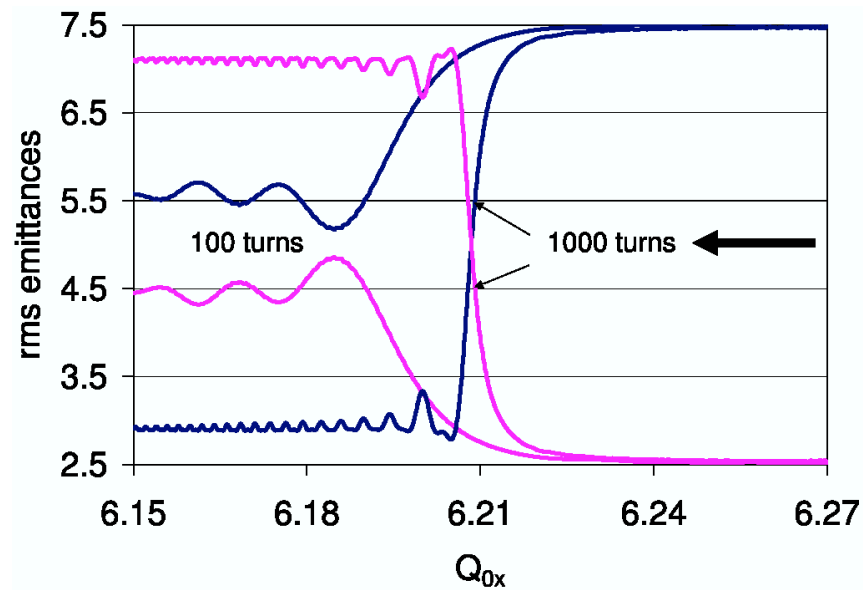

FIG. 20. (Color) Same as Fig. 19 with crossing from above (100 and 1000 turns).
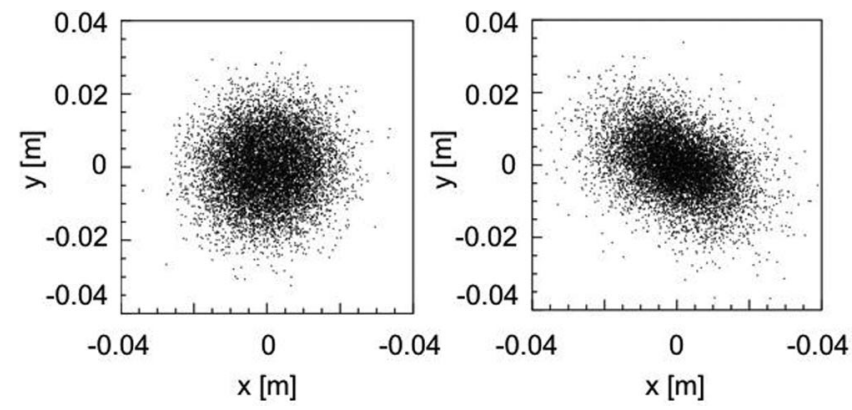

FIG. 21. Projection onto $x-y$ plane at $Q_{0, x}=6.21$; crossing from below (left frame) and from above (right frame) over 1000 turns.

the zero-space-charge theoretical modelling of skew exchange as derived in Ref. [13].

The same scaling invariance of all graphs with $\Delta Q_{x}$ is found as in Sec. IV C, if the skew strength is also multiplied by $\alpha$.

The fundamental difference in the crossing from below and above is also seen by analyzing the scatter plots at the symmetry point $Q_{0, x}=6.21$ shown in Fig. 21 for the 1000 turns cases.

The crossing from below shows practically the same result as without skew: no correlation in seen in $x-y$, whereas in the $x-y^{\prime}$ plane the same pronounced fourfold correlation pattern is found as in Fig. 11, which confirms that the exchange is entirely controlled by the Montague fourth order space-charge effect. For the crossing from above, instead, one finds a correlation in real space in terms of a $45^{\circ}$ rotation of the beam. Such a physical rotation (on resonance) is the typical behavior for linear coupling in the absence of space charge. This rotation also explains the above described enhancement effect by space charge: the space-charge self-skewing is an instability process, which requires an initial start-here induced by the external skew rotation - to become effective.

The case of split tunes with skew quadrupoles and space charge is studied in Ref. [9]. It is controlled by the external linear coupling - modified by space charge, while selfskewing and Montague resonance effects are absent. The crossing from below results in a similar smooth and spacecharge dominated emittance exchange as in the present study. The crossing from above, however, shows a discontinuous feature, independent of how slow the crossing is. This is explained again by the observation of stop-band attraction: the starting emittance coupling quickly pushes the stop band over the working-point, and the exchange ceases.

\section{CONCLUSION}

The space-charge-driven "Montague" coupling resonance reveals collective and nonlinear behavior, for which emittance-dependent detuning caused by space charge plays an important role. We have shown that a comparison 
with the unstable mode spectra from the analytical theory of KV-beams allows quantifying this detuning and the associated stop bands and growth rates. Space charge and the emittance-dependent detuning determine the lower edge of the stop band (for $\epsilon_{r}>1$, otherwise reversed); whereas the upper edge is given by the fixed condition $Q_{0, x}=Q_{0, y}$. We find that this asymmetry also gives rise to a strong directionality for dynamical resonance crossing, with adiabatic and reversible behavior for slow crossing from below, but discontinuous behavior for crossing from above (repulsive, respectively, attractive edges). The discontinuous behavior can be removed by a sufficiently large linear coupling, which makes the crossing from above entirely skew dominated, whereas the crossing from below remains space-charge dominated. In future work it should be explored how this asymmetry behaves, if additional time-dependent and possibly irreversible effects are included, like intrabeam scattering or synchrotron motion. One may also expect that the memory effects in the correlated phase space, which lead to adiabatic and reversible behavior, get washed out if the crossing occurs in a time much longer than the intrabeam scattering or synchrotron oscillation time scale. Preliminary simulations shown in Ref. [14] give some evidence for such a memory extinction.

[1] B. W. Montague, CERN-Report No. 68-38, CERN, 1968.
[2] P. Spiller, Proceedings of the Particle Accelerator Conference, Knoxville, 2005, p. 294.

[3] E. Metral et al., AIP Conf. Proc. 773, 122 (2005).

[4] I. Hofmann et al., Proceedings of the Particle Accelerator Conference, Knoxville, 2005, p. 330.

[5] I. Hofmann, G. Franchetti, J. Qiang, R. Ryne, F. Gerigk, D. Jeon, and N. Pichoff, Proceedings of the European Accelerator Conference, Paris, 2002, edited by J.L. Laclare (2002), p. 74.

[6] I. Hofmann and O. Boine-Frankenheim, Phys. Rev. Lett. 87, 034802 (2001).

[7] I. Hofmann, G. Franchetti, O. Boine-Frankenheim, J. Qiang, and R. D. Ryne, Phys. Rev. ST Accel. Beams 6, 024202 (2003).

[8] G. Franchetti, I. Hofmann, and G. Turchetti, AIP Conf. Proc. 448, 233 (1998).

[9] G. Franchetti, I. Hofmann, and M. Aslaninejad, Phys. Rev. Lett. 94, 194801 (2005).

[10] A. J. Dragt, F. Neri, and G. Rangarajan, Phys. Rev. A 45, 2572 (1992).

[11] R. A. Kishek, J. J. Barnard, and D. P. Grote, IEEE Particle Accelerator Conference (PAC 99), New York, 1999, p. 1761 .

[12] I. Hofmann, Phys. Rev. E 57, 4713 (1998).

[13] E. Metral, Technical Report No. CERN-PS-2001-066-AE, CERN (unpublished).

[14] I. Hofmann, G. Franchetti, J. Qiang, and R. Ryne, Proceedings of the European Accelerator Conference, Luzern, 2004, edited by L. Rivkin (EPS-AG, Geneva, 2004), p. 1960. 